\title{
A Study on Perception and knowledge regarding HIV/AIDS among school students and significance of educational intervention Dhungel BA ${ }^{1}$, Chaudhary $\mathbf{M}^{2}$, Singh $S^{3}$, Dhungel KU ${ }^{1}$
}

${ }^{1}$ Researcher, Think Tank Foundation, Jorpati, Nepal

${ }^{2}$ Department of Microbiology, KIST Medical College, Kathmandu, Nepal

${ }^{3}$ Department of Biochemistry, KIST Medical College, Kathmandu, Nepal

\begin{abstract}
Background and objectives: HIV/AIDS is a burning crisis worldwide. Cure of HIV is not yet possible so prevention is of prime importance in control of HIV. Education plays an important role in bringing awareness. This study is planned to find the existing knowledge and significance of educational intervention.

Material and Methods: This study was an experimental design in which same group of subjects (students to class 9 and 10) of selected schools in Imadol, served as control and experimental group. Participants' perception and knowledge about various aspects of HIV/AIDS were studied by noting their agreement with a set of 65 statements (questionnaire) using a modified Likert-type scale. The data was entered in SPSS. A p value less than 0.05 were taken as statistically significant.
\end{abstract}

Result: In this study, educational intervention has proved statistically significant in public school ( $\mathrm{p}$ value 0.02 ). Among different sub groups total median score for knowledge about HIV/AIDS and idea about vulnerable group was also statistically significant ( $p$ value 0.01 ).

Conclusion:Educational intervention does play significant role in strengthening knowledge and perception regarding issues related to HIV/AIDS.

Key words: HIV, AIDS, Educational intervention, Knowledge

\section{INRODUCTION}

HIV/AIDS is a burning crisis worldwide [1]. When it hits a least developed nation like Nepal, management of HIV/AIDS becomes even more difficult. The first case of HIV was detected in 1988 AD in Nepal and by 2012AD $39,000-65,000$ people are estimated to be living with HIV [2]. Given the existing medical and public health reporting system in Nepal, it is very likely that the actual number of cases is many times higher than the documented cases [3]. Nepal has moved from low prevalence status to concentrated epidemics with prevalence of $17.3 \%$ and $68 \%$ among female sex workers and intravenous drug users respectively [4]. There is evidence of explosive increase in the number of 
infection since 1996 [5]. AIDS is a leading cause of death in 15-49 years age group, which means 100,000-200,000 young adults are likely to become infected and overall 10,000-15,000 new annual AIDS cases due to AIDS related illness. This will further increase the poverty, vulnerability and overall negative impact on socioeconomic development of the nation [4].

The country's vulnerability to HIV/AIDS is exacerbated by geographic and ethnic diversity. Its poverty, inequality, underdevelopment, civil conflict, political instability, migration abroad for work and varied level of knowledge about HIV transmission among most at risk group and young population. Nepal is confronted with increasing incidence of HIV among young adults[6]. Injecting drug users are one of the most susceptible groups to acquire HIV. Adolescent are the identified vulnerable group for intravenous drug users. Prevention intervention coverage is low for people under 25years of age. There is need to ensure that $80 \%$ of young people who are the most at risk are reached with correct information and acquire appropriate skills and knowledge for HIV prevention[7].

Nepal has inadequate infrastructure such as clinics, district hospitals and distribution units needed to provide antiretroviral treatment for people living with HIV [4]. This further signifies the need and importance of prevention activities. Prevention is also much cheaper to implement than curative means especially in a developing country like Nepal.

Although adolescents in Kathmandu have some information regarding HIV/AIDS, there are still some misconceptions in some key areas of knowledge and perception. Many have not encountered people with HIV. This situation may lead to unnecessary fear, ignorance and uncertainty among adolescents. They may also develop negative attitude towards HIV positive people due to over emphasis of the dreadfulness of HIV infection. It may produce irrational behavior in adolescents toward those with HIV/AIDS. In Nepal, there are limited school education program on sexually transmitted disease including HIV/AIDS. Students' theoretical knowledge, even good enough regarding the virus that can cause AIDS may not be sufficient to make them practically more sound regarding the issues related to HIV/AIDS. They need to realize the fact that young people like them can get HIV if they are into some risk taking activities like sharing needles[8]. Their sensitivity towards people living with HIV/AIDS need to accommodate the fact that HIV positive children can be born from HIV positive mother and these children have equal right to live a normal life, go to normal school and mingle with other healthy kids. Hence, this study was planned and carried out to assess the perception, knowledge and awareness of adolescents regarding HIV/AIDS and to identify the areas of confusion that might serve as an important target of educational intervention. This study had three objectives-

1. To find out the existing knowledge participants had regarding HIV/AIDS

2. To impart educational intervention

3. To find out the effect of educational interventional in participants' knowledge about HIV/AIDS and to opinion towards people living with HIV/AIDS. 


\section{MATERIALS AND METHODS}

This study was an experimental design in which same group of subjects served as control and experimental group. The study was carried among school students (classes 9 and 10) in Imadol, Lalitpur. Participants were explained about the aims and objectives of the study and invited to participate. Written informed consent was obtained from all participants.

Basic demographic information like gender, age, ethnic/caste group, number of family members were collected. Participants' perception ad knowledge about various aspects of HIV/AIDS were studied by noting their agreement with a set of 65 statements (questionnaire) using a modified Likert-type scale. The questionnaire was designed after proper literature review and with the consultation with experts in English and Nepali both languages. The two questionnaires (in both languages) were compared by two individuals conversant in both English and Nepali and not involved in the study. Participants could attempt either the English or the Nepali version of the questionnaire according to their preference.

A set of questionnaire was distributed to students as a pretest. After analysis of the response in pretest, the educational session was designed. The session was full of interaction, discussion and talk from expert. Post test was taken immediately after the educational session. Before the final study, pilot study was done in one school.

Selection criteria of the study subjects: Students of class 9 and 10 in the selected school
Subjects (sample size): Number of students present at the time.

Statistical analysis: The data was entered in SPSS. The score of negative statements was reversed while calculating the final score. The median scores of individual statements were calculated to know areas in which respondents' perception is low.

The median total score and the median scores of various subcategories calculated. The scores were compared among various subgroups (as different ethnic group, age group etc) of respondents using appropriate non-parametric tests. A p value less than 0.05 was taken as statistically significant.

The post-session scores was compared to those before the session (pre-session scores), appropriate non-parametric tests will be used. Suitable table and diagram are projected.

As participant identity was not noted so we could not measure the change in individual participant's scores before and after the session. Scores was hence compared using non-parametric tests for independent samples.

\section{Parameters of the research:}

\section{A. Knowledge about HIV/AIDS}

General awareness about HIV/AIDS

Educational tools for HIV/AIDS

Knowledge about transmission and misconception related to transmission of HIV/AIDS Knowledge about vulnerability group Knowledge regarding prevention Knowledge regarding treatment

\section{B. Perception about HIV/AIDS: Sensitivity and opinion related to}


issues about people living with HIV/AIDS

\section{Other Study parameters were}

Gender, Family members, Mother's occupation, Public/Private school

\section{Tools/ machines: Questionnaire} with details about the students and 65 set of statements using Likert scale was used as tools. To reduce stereotyped responses certain statements were negative. The score of negative statements were reversed while calculating the final score. The statements dealt with issues like knowledge among participants regarding HIV transmission, vulnerability, treatment, prevention and perception towards people living with HIV/AIDS.

\section{RESULTS}

The study was carried out in public and private schools selected in Imadol, Lalitpur. The demographic details about the participants of the two groups are presented in Table 1 and 2.

\begin{tabular}{|l|l|l|l|l|}
\hline \multicolumn{5}{|c|}{$\begin{array}{l}\text { Table 1: Demographic characteristics of respondents } \\
\text { before and after the educational session in public schools }\end{array}$} \\
\cline { 2 - 5 } $\begin{array}{l}\text { Demographic } \\
\text { feature }\end{array}$ & \multicolumn{2}{|c|}{ Pre-test } & \multicolumn{2}{c|}{ Post-test } \\
\hline Gender & & & & N \\
Male & 16 & $32 \%$ & 16 & $33 \%$ \\
Female & 34 & $68 \%$ & 32 & $67 \%$ \\
\hline Ethnic group & & & & \\
Brahmin/Chhetri & 30 & $60 \%$ & 30 & $63 \%$ \\
Newar & 8 & $16 \%$ & 8 & $16 \%$ \\
Others & 12 & $24 \%$ & 10 & $1 \%$ \\
\hline No. of family & & & & \\
members & & & & \\
\hline$<4$ & 00 & $00 \%$ & 00 & $00 \%$ \\
$4-6$ & 44 & $88 \%$ & 42 & $88 \%$ \\
$7-10$ & 06 & $12 \%$ & 06 & $12 \%$ \\
\hline Mother's & \multicolumn{4}{|l}{} \\
occupation & 48 & $96 \%$ & 46 & $96 \%$ \\
Home maker & 02 & $04 \%$ & 02 & $04 \%$ \\
Others & \multicolumn{4}{|l}{} \\
\hline
\end{tabular}

$\mathrm{N}$; Number, \%, percentage

\begin{tabular}{|l|l|l|l|c|}
\hline $\begin{array}{l}\text { Table 2: Demographic characteristics of respondents } \\
\text { before and after the educational session in private } \\
\text { schools }\end{array}$ \\
\begin{tabular}{|l|c|c|c|} 
Demographic \\
feature
\end{tabular} & \multicolumn{2}{|c|}{ Pre-test } & \multicolumn{2}{c|}{ Post-test } \\
\cline { 2 - 5 } & $\mathrm{N}$ & $\%$ & $\mathrm{~N}$ & $\%$ \\
\hline Gender & & & & \\
Male & 32 & $47 \%$ & 32 & $50 \%$ \\
Female & 36 & $53 \%$ & 32 & $50 \%$ \\
\hline Ethnic group & & & & \\
Brahmin/Chhetri & 30 & $44 \%$ & 28 & $44 \%$ \\
Newar & 14 & $16 \%$ & 14 & $22 \%$ \\
Others & 24 & $40 \%$ & 22 & $34 \%$ \\
\hline No. of family & & & & \\
members & & & & \\
$<4$ & 02 & $03 \%$ & 02 & $03 \%$ \\
4-6 & 60 & $88 \%$ & 57 & $89 \%$ \\
$7-10$ & 06 & $09 \%$ & 05 & $08 \%$ \\
\hline Mother's & & & & \\
occupation & & & & \\
Home maker & 66 & $97 \%$ & 62 & $97 \%$ \\
Others & 02 & $03 \%$ & 02 & $03 \%$ \\
\hline
\end{tabular}

$\mathrm{N}$; Number, \%, percentage

Total median score before and after the educational intervention among the students in public and private schools are presented in table 3 and 4 .

\begin{tabular}{|c|c|c|}
\hline Statement & Before & After \\
\hline ONE (major problem) & 5 & 5 \\
\hline TWO (easy access counseling centre) & 4 & 5 \\
\hline THREE (caused by virus) & 5 & 5 \\
\hline $\begin{array}{l}\text { FOUR (common only in certain risk } \\
\text { group people) }\end{array}$ & 1 & 1 \\
\hline FIVE(need of sex education in school) & 4 & 5 \\
\hline SIX( HIV/AIDS-life threatening disease) & 5 & 5 \\
\hline $\begin{array}{l}\text { SEVEN(should not hesitate to ask } \\
\text { queries about HIV) }\end{array}$ & 3 & 3 \\
\hline $\begin{array}{l}\text { EIGHT(difficult to approach teachers to } \\
\text { ask queries) }\end{array}$ & 1 & 1 \\
\hline $\begin{array}{l}\text { NINE(friends are reliable source of } \\
\text { information) }\end{array}$ & 4 & 4 \\
\hline $\begin{array}{l}\text { TEN (should not ask family, the queries } \\
\text { about HIV) }\end{array}$ & 3 & 3 \\
\hline $\begin{array}{l}\text { ELEVEN((can visit doctors to ask } \\
\text { queries) }\end{array}$ & 5 & 5 \\
\hline $\begin{array}{l}\text { TWELVE(Public announcement as best } \\
\text { source of information) }\end{array}$ & 4 & 4 \\
\hline $\begin{array}{l}\text { THIRTEEN(Hoarding boards should be } \\
\text { kept at many places) }\end{array}$ & 4 & 4 \\
\hline $\begin{array}{l}\text { FOURTEEN(Newspapers/health } \\
\text { magazines as easy source of } \\
\text { information) }\end{array}$ & 4 & 4 \\
\hline $\begin{array}{l}\text { FIFTEEN(TV/Radio as source of } \\
\text { information) }\end{array}$ & 4 & 5 \\
\hline SIXTEEN(sexually transmitted disease) & 5 & 5 \\
\hline $\begin{array}{l}\text { SEVENTEEN(can be transmitted by } \\
\text { vertical transmission) }\end{array}$ & 5 & 5 \\
\hline $\begin{array}{l}\text { EIGHTEEN(cannot be transmitted from } \\
\text { breast milk) }\end{array}$ & 3 & 2 \\
\hline NINTEEN(disease of homosexuals only) & 1 & 1 \\
\hline TWENTY(instrument used in barber & 1.5 & 4 \\
\hline
\end{tabular}




\begin{tabular}{|c|c|c|}
\hline $\begin{array}{l}\text { shop as a probable source of } \\
\text { transmission) }\end{array}$ & & \\
\hline $\begin{array}{l}\text { TWENTY ONE(HIV positive can donate } \\
\text { blood) }\end{array}$ & 1.5 & 1 \\
\hline $\begin{array}{l}\text { TWENTY TWO(condom can prevent } \\
\text { HIV) }\end{array}$ & 2 & 5 \\
\hline $\begin{array}{l}\text { TWENTY THREE(can be transmitted } \\
\text { through syringe) }\end{array}$ & 3 & 5 \\
\hline $\begin{array}{l}\text { TWENTY FOUR(HIV can be transmitted } \\
\text { in window period) }\end{array}$ & 3 & 3 \\
\hline $\begin{array}{l}\text { TWENTY FIVE(can be transmitted by } \\
\text { insect bite) }\end{array}$ & 3 & 1 \\
\hline $\begin{array}{l}\text { TWENTY SIX(cannot be transmitted } \\
\text { by lip kissing) }\end{array}$ & 3 & 4 \\
\hline $\begin{array}{l}\text { TWENTY SEVEN(transmitted by } \\
\text { sharing toilet with infected person) }\end{array}$ & 2 & 1 \\
\hline $\begin{array}{l}\text { TWENTY EIGHT(sharing meal with } \\
\text { HIV positive is dangerous) }\end{array}$ & 3 & 1 \\
\hline $\begin{array}{l}\text { TWENTY NINE(transmitted by sharing } \\
\text { cloth with infected person) }\end{array}$ & 1 & 1 \\
\hline $\begin{array}{l}\text { THIRTY(transmitted by hugging } \\
\text { infected person) }\end{array}$ & 1 & 1 \\
\hline $\begin{array}{l}\text { THIRTY ONE(illiterate females are at } \\
\text { high risk) }\end{array}$ & 3 & 4 \\
\hline $\begin{array}{l}\text { THIRTY TWO(person with STDs are at } \\
\text { high risk) }\end{array}$ & 5 & 5 \\
\hline THIRTY THREE(IVDUs are at high risk) & 5 & 5 \\
\hline $\begin{array}{l}\text { THIRTY FOUR(people of any socio } \\
\text { economic status can get HIV) }\end{array}$ & 5 & 5 \\
\hline $\begin{array}{l}\text { THIRTY FIVE(youth are at high risk } \\
\text { of getting HIV) }\end{array}$ & 3 & 4 \\
\hline $\begin{array}{l}\text { THIRTY SIX(Prostitutes are at high } \\
\text { risk) }\end{array}$ & 3 & 4 \\
\hline $\begin{array}{l}\text { THIRTY SEVEN(alcoholics are at high } \\
\text { risk of getting HIV) }\end{array}$ & 5 & 5 \\
\hline THIRTY EIGHT (any caste can get HIV) & 5 & 5 \\
\hline $\begin{array}{l}\text { THIRTY NINE(People of any age can get } \\
\text { HIV) }\end{array}$ & 5 & 5 \\
\hline $\begin{array}{l}\text { FORTY(Multiple sex partners } \\
\text { increases the chance of HIV } \\
\text { infection) }\end{array}$ & 3 & 5 \\
\hline $\begin{array}{l}\text { FORTY ONE(Migrant laborers are at } \\
\text { high risk) }\end{array}$ & 4 & 5 \\
\hline $\begin{array}{l}\text { FORTY TWO(Co infection of HIV and } \\
\text { TB increases the severity of both } \\
\text { disease) }\end{array}$ & 3 & 5 \\
\hline $\begin{array}{l}\text { FORTY THREE(I would stop being } \\
\text { friend with HIV infected person) }\end{array}$ & 3 & 2 \\
\hline $\begin{array}{l}\text { FORTY FOUR(Young people with HIV } \\
\text { should be allowed to study in school. }\end{array}$ & 4 & 5 \\
\hline $\begin{array}{l}\text { FORTY FIVE(People with HIV should } \\
\text { not be allowed to work in office with } \\
\text { others) }\end{array}$ & 3.5 & 1 \\
\hline $\begin{array}{l}\text { FORTY SIX(I am afraid of people with } \\
\text { HIV/AIDS) }\end{array}$ & 1 & 1 \\
\hline $\begin{array}{l}\text { FORTY SEVEN(Hands should not be } \\
\text { shaked with HIV infected people) }\end{array}$ & 1 & 1 \\
\hline $\begin{array}{l}\text { FORTY EIGHT(HIV is transmitted by } \\
\text { sharing materials at office) }\end{array}$ & 1 & 1 \\
\hline $\begin{array}{l}\text { FORTY NINE(HIV can be transmitted } \\
\text { by cough/sneeze,) }\end{array}$ & 3 & 1 \\
\hline $\begin{array}{l}\text { FIFTY(Knowledge helps in } \\
\text { prevention) }\end{array}$ & 4 & 5 \\
\hline $\begin{array}{l}\text { FIFTY ONE(Avoidance of physical } \\
\text { relation helps in prevention) }\end{array}$ & 3 & 4 \\
\hline $\begin{array}{l}\text { FIFTY TWO (poor cannot afford } \\
\text { treatment) }\end{array}$ & 3 & 4 \\
\hline
\end{tabular}

\begin{tabular}{|l|l|l|}
\hline $\begin{array}{l}\text { FIFTY THREE(contraceptives can } \\
\text { prevent HIV) }\end{array}$ & $\mathbf{3}$ & $\mathbf{3}$ \\
\hline FIFTY FOUR(drugs can cure HIV) & $\mathbf{3}$ & $\mathbf{2}$ \\
\hline $\begin{array}{l}\text { FIFTY FIVE(HAART is effective } \\
\text { treatment for HIV) }\end{array}$ & $\mathbf{3}$ & $\mathbf{4}$ \\
\hline $\begin{array}{l}\text { FIFTY SIX(Washing of genitals after } \\
\text { sexual intraction helps to reduce } \\
\text { chance of HIV) }\end{array}$ & 3 & 4 \\
\hline $\begin{array}{l}\text { FIFTY SEVEN(CD } \\
\text { marker in treating HIV) }\end{array}$ & $\mathbf{3}$ effective & $\mathbf{4}$ \\
\hline $\begin{array}{l}\text { FIFTY EIGHT(People with HIV should } \\
\text { get proper counceling) }\end{array}$ & $\mathbf{3}$ & $\mathbf{5}$ \\
\hline $\begin{array}{l}\text { FIFTY NINE(Western blot confirms } \\
\text { infection with HIV) }\end{array}$ & $\mathbf{3}$ & $\mathbf{4}$ \\
\hline SIXTY(I will care people with HIV) & 4 & 4 \\
\hline SIXTY ONE(Test can detect HIV) & 3 & 5 \\
\hline $\begin{array}{l}\text { SIXTY TWO(People with HIV are only to } \\
\text { blame themselves) }\end{array}$ & 4 & 4 \\
\hline $\begin{array}{l}\text { SIXTY THREE(I have sympathy for } \\
\text { people with HIV) }\end{array}$ & $\mathbf{3}$ & $\mathbf{4}$ \\
\hline $\begin{array}{l}\text { SIXTY FOUR(I dislike people with } \\
\text { HIV) }\end{array}$ & $\mathbf{2}$ & $\mathbf{1}$ \\
\hline $\begin{array}{l}\text { SIXTY FIVE(I can protect myself from } \\
\text { acquiring HIV) }\end{array}$ & $\mathbf{3}$ & $\mathbf{5}$ \\
\hline
\end{tabular}

Total median score before and after the educational intervention showed statistical significance ( $\mathrm{p}$ value 0.02 ) among students of public school (table 3) whereas no statistical significance ( $\mathrm{p}$ value 0.15 ) was seen among students of private school (table 4).

\begin{tabular}{|l|l|l|}
\hline $\begin{array}{l}\text { Table 4: Median Scores of individual statements before } \\
\text { and after educational session in private schools }\end{array}$ \\
\hline Statement & Before & After \\
\hline ONE (major problem) & 5 & 5 \\
\hline $\begin{array}{l}\text { TWO (easy access counseling } \\
\text { centre) }\end{array}$ & $\mathbf{3 . 5}$ & $\mathbf{5}$ \\
\hline THREE (caused by virus) & 4 & 5 \\
\hline $\begin{array}{l}\text { FOUR (common only in certain risk } \\
\text { group people) }\end{array}$ & $\mathbf{2}$ & $\mathbf{5}$ \\
\hline FIVE(need of sex education in school) & 2 & 1.5 \\
\hline $\begin{array}{l}\text { SIX( HIV/AIDS-life threatening } \\
\text { disease) }\end{array}$ & 4.5 & 5 \\
\hline $\begin{array}{l}\text { SEVEN(should not hesitate to ask } \\
\text { queries about HIV) }\end{array}$ & 4 & 4 \\
\hline $\begin{array}{l}\text { EIGHT(difficult to approach } \\
\text { teachers to ask queries) }\end{array}$ & $\mathbf{4}$ & $\mathbf{4}$ \\
\hline $\begin{array}{l}\text { NINE(friends are reliable source of } \\
\text { information) }\end{array}$ & 2.5 & 2 \\
\hline $\begin{array}{l}\text { TEN (should not ask family, the } \\
\text { queries about HIV) }\end{array}$ & $\mathbf{5}$ & $\mathbf{5}$ \\
\hline $\begin{array}{l}\text { ELEVEN((can visit doctors to ask } \\
\text { queries) }\end{array}$ & 5 & 5 \\
\hline $\begin{array}{l}\text { TWELVE(Public announcement as } \\
\text { best source of information) }\end{array}$ & $\mathbf{3}$ & $\mathbf{5}$ \\
\hline $\begin{array}{l}\text { THIRTEEN(Hoarding boards should be } \\
\text { kept at many places) }\end{array}$ & 4 & 4 \\
\hline $\begin{array}{l}\text { FOURTEEN(Newspapers/health } \\
\text { magazines as easy source of } \\
\text { information) }\end{array}$ & $\mathbf{2}$ & $\mathbf{4}$ \\
\hline
\end{tabular}




\begin{tabular}{|c|c|c|}
\hline $\begin{array}{l}\text { FIFTEEN(TV/Radio as source of } \\
\text { information) }\end{array}$ & 1 & 1 \\
\hline $\begin{array}{l}\text { SIXTEEN(sexually transmitted } \\
\text { disease) }\end{array}$ & 5 & 5 \\
\hline $\begin{array}{l}\text { SEVENTEEN(can be transmitted by } \\
\text { vertical transmission) }\end{array}$ & 2 & 4 \\
\hline $\begin{array}{l}\text { EIGHTEEN(cannot be transmitted } \\
\text { from breast milk) }\end{array}$ & 2 & 1 \\
\hline $\begin{array}{l}\text { NINTEEN(disease of homosexuals } \\
\text { only) }\end{array}$ & 4 & 2 \\
\hline $\begin{array}{l}\text { TWENTY(instrument used in barber } \\
\text { shop as a probable source of } \\
\text { transmission) }\end{array}$ & 3.5 & 4 \\
\hline \multirow{2}{*}{$\begin{array}{l}\text { TWENTY ONE(HIV positive can } \\
\text { donate blood) }\end{array}$} & 5 & 1 \\
\hline & 3.5 & 1.5 \\
\hline $\begin{array}{l}\text { TWENTY THREE(can be transmitted } \\
\text { through syringe) }\end{array}$ & 2 & 5 \\
\hline $\begin{array}{l}\text { TWENTY FOUR(HIV can be } \\
\text { transmitted in window period) }\end{array}$ & 4 & 5 \\
\hline $\begin{array}{l}\text { TWENTY FIVE(can be transmitted } \\
\text { by insect bite) }\end{array}$ & 3 & 1 \\
\hline $\begin{array}{l}\text { TWENTY SIX(cannot be transmitted by } \\
\text { lip kissing) }\end{array}$ & 2 & 2 \\
\hline $\begin{array}{l}\text { TWENTY SEVEN(transmitted by } \\
\text { sharing toilet with infected person) }\end{array}$ & 2 & 1 \\
\hline $\begin{array}{l}\text { TWENTY EIGHT(sharing meal with } \\
\text { HIV positive is dangerous) }\end{array}$ & 2 & 1 \\
\hline $\begin{array}{l}\text { TWENTY NINE(transmitted by sharing } \\
\text { cloth with infected person) }\end{array}$ & 1 & 1 \\
\hline $\begin{array}{l}\text { THIRTY(transmitted by hugging } \\
\text { infected person) }\end{array}$ & 5 & 1 \\
\hline $\begin{array}{l}\text { THIRTY ONE(illiterate females are at } \\
\text { high risk) }\end{array}$ & 4.5 & 5 \\
\hline $\begin{array}{l}\text { THIRTY TWO(person with STDs are } \\
\text { at high risk) }\end{array}$ & 3 & 4 \\
\hline $\begin{array}{l}\text { THIRTY THREE(IVDUs are at high } \\
\text { risk) }\end{array}$ & 5 & 5 \\
\hline $\begin{array}{l}\text { THIRTY FOUR(people of any socio } \\
\text { economic status can get HIV) }\end{array}$ & 4 & 4 \\
\hline $\begin{array}{l}\text { THIRTY FIVE(youth are at high risk } \\
\text { of getting HIV) }\end{array}$ & 3 & 4 \\
\hline $\begin{array}{l}\text { THIRTY SIX(Prostitutes are at high } \\
\text { risk) }\end{array}$ & 3 & 5 \\
\hline $\begin{array}{l}\text { THIRTY SEVEN(alcoholics are at high } \\
\text { risk of getting HIV) }\end{array}$ & 2 & 2 \\
\hline THIRTY EIGHT (any caste can get HIV) & 5 & 5 \\
\hline $\begin{array}{l}\text { THIRTY NINE(People of any age can } \\
\text { get HIV) }\end{array}$ & 4 & 5 \\
\hline $\begin{array}{l}\text { FORTY(Multiple sex partners } \\
\text { increases the chance of HIV } \\
\text { infection) }\end{array}$ & 3 & 2 \\
\hline $\begin{array}{l}\text { FORTY ONE(Migrant laborers are at } \\
\text { high risk) }\end{array}$ & 4 & 4 \\
\hline $\begin{array}{l}\text { FORTY TWO(Co infection of HIV and } \\
\text { TB increases the severity of both } \\
\text { disease) }\end{array}$ & 1 & 4 \\
\hline $\begin{array}{l}\text { FORTY THREE(I would stop being } \\
\text { friend with HIV infected person) }\end{array}$ & 3 & 3 \\
\hline $\begin{array}{l}\text { FORTY FOUR(Young people with } \\
\text { HIV should be allowed to study in } \\
\text { school. }\end{array}$ & 1 & 4 \\
\hline $\begin{array}{l}\text { FORTY FIVE(People with HIV should } \\
\text { not be allowed to work in office with } \\
\text { others) }\end{array}$ & 1 & 1 \\
\hline $\begin{array}{l}\text { FORTY SIX(I am afraid of people with } \\
\text { HIV/AIDS) }\end{array}$ & 1.5 & 1.5 \\
\hline
\end{tabular}

\begin{tabular}{|l|l|l|}
\hline $\begin{array}{l}\text { FORTY SEVEN(Hands should not be } \\
\text { shaked with HIV infected people) }\end{array}$ & 1 & 1 \\
\hline $\begin{array}{l}\text { FORTY EIGHT(HIV is transmitted by } \\
\text { sharing materials at office) }\end{array}$ & 2 & 1 \\
\hline $\begin{array}{l}\text { FORTY NINE(HIV can be transmitted } \\
\text { by cough/sneeze, })\end{array}$ & 1 & 1 \\
\hline $\begin{array}{l}\text { FIFTY(Knowledge helps in } \\
\text { prevention) }\end{array}$ & $\mathbf{5}$ & $\mathbf{5}$ \\
\hline $\begin{array}{l}\text { FIFTY ONE(Avoidance of physical } \\
\text { relation helps in prevention) }\end{array}$ & $\mathbf{2}$ & $\mathbf{4 . 5}$ \\
\hline $\begin{array}{l}\text { FIFTY TWO (poor cannot afford } \\
\text { treatment) }\end{array}$ & $\mathbf{2}$ & $\mathbf{4}$ \\
\hline $\begin{array}{l}\text { FIFTY THREE(contraceptives can } \\
\text { prevent HIV) }\end{array}$ & $\mathbf{3}$ & $\mathbf{1}$ \\
\hline FIFTY FOUR(drugs can cure HIV) & $\mathbf{3}$ & $\mathbf{2}$ \\
\hline $\begin{array}{l}\text { FIFTY FIVE(HAART is effective } \\
\text { treatment for HIV) }\end{array}$ & $\mathbf{2}$ & $\mathbf{4}$ \\
\hline $\begin{array}{l}\text { FIFTY SIX(Washing of genitals after } \\
\text { sexual intraction helps to reduce } \\
\text { chance of HIV) }\end{array}$ & $\mathbf{3}$ & $\mathbf{4}$ \\
\hline $\begin{array}{l}\text { FIFTY SEVEN(CD } \text { count is effective } \\
\text { marker in treating HIV) }\end{array}$ & 4 & 4 \\
\hline $\begin{array}{l}\text { FIFTY EIGHT(People with HIV } \\
\text { should get proper counseling) }\end{array}$ & $\mathbf{3}$ & $\mathbf{4}$ \\
\hline $\begin{array}{l}\text { FIFTY NINE(Western blot confirms } \\
\text { infection with HIV) }\end{array}$ & 4 & 5 \\
\hline SIXTY(I will care people with HIV) & $\mathbf{2 . 5}$ & $\mathbf{4}$ \\
\hline SIXTY ONE(Test can detect HIV) & $\mathbf{3 . 5}$ & $\mathbf{4}$ \\
\hline $\begin{array}{l}\text { SIXTY TWO(People with HIV are only } \\
\text { to blame themselves) }\end{array}$ & 4 & 4 \\
\hline $\begin{array}{l}\text { SIXTY THREE(I have sympathy for } \\
\text { people with HIV) }\end{array}$ & $\mathbf{1 . 5}$ & $\mathbf{4}$ \\
\hline $\begin{array}{l}\text { SIXTY FOUR(I dislike people with } \\
\text { HIV) }\end{array}$ & $\mathbf{4}$ & $\mathbf{2}$ \\
\hline $\begin{array}{l}\text { SIXTY FIVE(I can protect myself } \\
\text { from acquiring HIV) }\end{array}$ & $\mathbf{4}$ & $\mathbf{4}$ \\
\hline
\end{tabular}

Among different subgroups, general knowledge regarding HIV/AIDS and knowledge about vulnerable group showed significant difference before and after the educational intervention at $\mathrm{p}<$ 0.01 . But, statistical significance was not found before and after the session about knowledge regarding transmission, opinion towards people living with HIV/AIDS and knowledge regarding prevention and treatment of HIV/AIDS.

\section{DISCUSSION}

Education plays an important role in bringing awareness in many issues. It is the best method for prevention of HIV/AIDS specially in a resource poor country like Nepal where treatment or 
care of people living with HIV/AIDS is still extremely difficult. Effective cure for HIV/AIDS is still not available and highly effective anti retroviral therapy against HIV/AIDS which reduces the viral growth and improves life of people with HIV are expensive and not easily available in far remote areas. The condition is exacerbating by the fact that many HIV cases are under diagnosed which may be due to lack of awareness and social inhibition. Many issues related to HIV needs educational intervention like imparting knowledge about prevention, treatment and attitude towards people living with HIV etc.

The developed world is dealing with issues of virology, immunology, and vaccines and anti retroviral drugs, we in the developing world still need to struggle for prevention of new infection and care of people living with HIV/ AIDS. Lack of adequate information about the virus and the myths and misconceptions surrounding it, the perceived risk of possible self infection, in addition to the widely held belief that people with HIV are "bad or immoral" generates stigma against people living with HIV/AIDS. It is known that education of the common people, enhancement of feelings of empathy and compassion, and economic empowerment of people living with HIV/AIDS are ways of minimizing the stigma.

The need of education for imparting knowledge regarding HIV/AIDS has been addressed to some extent by academic curriculum of school at higher secondary level. However, most of the issues taught only emphasize on theoretical knowledge and students are left unanswered to their practical life based queries related to the topic. This is reflected in our study also where students have presented that they hesitate to ask queries, are not comfortable to ask queries to family. They are more comfortable to discuss about these sensitive issues to their friends but the information collected from friends may not be reliable and it can mislead youth. So, more practical life based educational content and teaching methodology can help solve the problem. In our study also educational intervention has proved statistically significant in public school ( $p$ value 0.02). The educational intervention was more like an open discussion and interaction where facilitators eased the situation by comfortably presenting and discussing many sensitive issue related to HIV which may have been considered social taboo. Students enthusiastically participated and asked their queries. So, we strongly feel that the open environment to discuss freely the queries that emerge in adolescent mind should be created in both school and home so that children do not seek unauthentic source for addressing their queries.

Among different sub groups total median score for knowledge about HIV/AIDS and idea about vulnerable group was statistically significant before and after educational intervention ( $p$ value 0.01). Major changes were noted in participants' knowledge about diagnosis using western blot, co infection and severity of tuberculosis and HIV in the case of co infection, chances of HIV in case of multiple sex partners.

In our study the total median score before and after educational intervention in private school does not show statistical significance ( $p$ value 0.15 ). This may be due to various reasons like exposure to knowledge, life style, opportunity to 
access health magazines and resources, teaching style and methodology in private school. This, however, needs to be verified by rigorous research which is not in the scope of this study. None the less, positive change in many aspects did show after educational intervention like students got aware that HIV can be transmitted from mother to child during pregnancy or delivery, HIV positive should never donate blood, HIV is not transmitted by insect bite or hugging infected people, contraceptives does not prevent HIV, test can detect $\mathrm{CD}_{4}$ count is important in prediction of HIV status, Western blot is important for confirmation of HIV infection. Their attitude towards people living with HIV/AIDS also changed after educational intervention like their opinion positively changed for allowing young people with HIV to study in school, they opinioned to have sympathy for people living with HIV.

\section{CONCLUSION}

Overall, our study showed that students at higher secondary level have some knowledge about HIV/AIDS but there are areas of misconception which can be addressed by educational intervention. Such study in a larger scale can help to identify the base line knowledge status at higher secondary level and design academic curriculum and teaching pedagogy accordingly.

\section{ACKNOWLEDGEMENT}

Our team would like to thank University Grants Commission, Nepal for taking a vital step to help us develop and contribute in the field of research. We would also like to thank KIST medical college for supporting us in our project. We like to thank the participants and school authority for their consent and support to carry out this study.

\section{REFERENCES}

1. Madkour, M.M., Saif, A.A., Shahed, M.A., Moutaery, K.R.A., Kudwah, A.A. Global epidemiology of Tuberculosis. Tuberculosis,2003; Springer.

2. UNAIDS. Report on global AIDS epidemic 2013. Retrieved from http://www.unaids.org/en/media/unaids/co ntentassets/documents/epidemiology/2013/ gr2013/UNAIDS Global Report 2013 en.pdf. accessed on September 3, 2013

3. Cheesebrough M. Medical laboratory manual for tropical countries. HIV supplement. 1990;3rd ed, ELBS 2(Suppl): S1-S23.

4. Park K. Concept of health and disease. Preventive and social medicine. 2002; $17^{\text {th }}$ ed. Banarsidas Bhanot.

5. Dhungel, B.A. Dhungel, K.U., Easow, J.M., Singh, Y.I. Opportunistic infection among HIV seropositive cases in Manipal Teaching Hospital, Pokhara. 2008; KUMJ, 6; 335-39

6. World Health Organization. A global emergency: a combined response. The world health report-2004. WHO 2004.

7. World Health Organization. Trends in health status. Health situation in the South-East Asia Region 2002; 1988-2000.

8. Dhungel, B.A. Dhungel, K.U., Easow, J.M., Singh, Y.I. Study of risk factors for HIV transmission among seropositive cases. 2006; JIOM, 28,3941 